\title{
Principais dificuldades do atendimento pré-hospitalar descritas pela produção cientifica nacional
}

\author{
Main difficulties of pre hospital care described by national scientific production
}

Principales dificultades de la atención pre hospitalaria descritas por la producción científica nacional

Maicon Douglas Xavier Braga ${ }^{1 *}$, Francielle Macedo Santos Ribeiro ${ }^{1}$, Stella Marys Braga Roque ${ }^{1}$, Fernanda Viana de Moraes $^{1}$, Laryssa Waleska Pereira de Santana ${ }^{1}$, Vilma Silva Lima².

\section{RESUMO}

Objetivo: Identificar por meio de uma revisão integrativa da literatura as principais dificuldades do atendimento pré-hospitalar descritas na produção científica nacional dos últimos 10 anos. Métodos: Trata-se de uma revisão integrativa da literatura realizada por meio de busca nas bases de dados Scielo e LILACS. Foram utilizados os descritores disponíveis no índice de descritores em ciências da saúde (DECS): serviço médico de emergência, urgência e emergência. Os critérios de inclusão utilizados foram artigos produzidos nos últimos 10 anos de origem nacional e em língua portuguesa, disponíveis nas bases de dados ou na plataforma do periódico e que respondiam à questão de pesquisa. Resultados: Após a busca na base de dados LILACS, utilizando-se os descritores serviço médico de emergência e emergências, foram encontrados 299 artigos, após aplicação dos filtros houve um resultado com 18 artigos que após leitura dos títulos e resumos resultou em uma amostra de 7 artigos. Na base de dados Scielo foram encontrados 56 artigos, após aplicação dos filtros houve um resultado com 17 artigos e com a leitura dos títulos e resumos foram considerados elegíveis 10 artigos para a amostra. Considerações finais: As principais dificuldades do atendimento pré-hospitalar descritas na produção científica nacional nos últimos 10 anos, observadas neste estudo, foram: falta de integração entre os serviços, demandas não pertinentes, déficit de materiais e falta de capacitação. Além disso, foram citadas também: a imobilidade na via pública, a exposição à violência urbana, a falha na comunicação entre os serviços e carência de protocolos específicos de atendimento.

Palavras-chave: Serviço médico de emergência, Urgência, Emergência.

\begin{abstract}
Objective: Aim to identify, through an integrative review of the literature, the main difficulties of prehospital care described in the national scientific production of the last 10 years. Methods: This is an integrative review of the literature by searching the SciELO and LILACS databases. The descriptors available in the index of descriptors in health sciences (DECS) used were: emergency medical service, urgency and emergency. The inclusion criteria used were articles produced in the last 10 years of national origin and in the Portuguese language, available in databases or on the journal platform, which answered the question of research. Results: After searching the LILACS database, using the descriptors emergency medical service and emergencies, we found 299 articles, after applying the filters there was a result with 18 articles that after reading the titles and summaries resulted in a sample of 7 articles. In the Scielo database, 56 articles were found, after application of the filters there was a result with 17 articles and the reading of the titles and abstracts were considered eligible 10 articles for the sample. Final considerations: The main difficulties of prehospital care described in
\end{abstract}

\footnotetext{
${ }^{1}$ Enfermeiros graduados pela Faculdade de Saúde Ibituruna (FASI). Montes Claros - MG.

*E-mail: maiconmoc2007@hotmail.com.

${ }^{2}$ Acadêmica do Curso de Psicologia da Faculdade de Saúde Ibituruna (FASI). Montes Claros - MG.
} 
the national scientific production in the last 10 years observed in this study were: lack of integration among services, non-pertinent demands, lack of materials and lack of capacity. Furthermore, were also mentioned: the immobility on the public road, the exposure to urban violence, the communication gap between services and the deficiency of specific care protocols.

Key-words: Medical services, Urgency, Emergency services.

\section{RESUMEN}

Objetivo:Identificar por medio de una revisión integrativa de la literatura las principales dificultades de la atención prehospitalaria descritas en la producción científica nacional de los últimos 10 años. Métodos: Se trata de una revisión integrativa de la literatura realizada por medio de búsqueda en las bases de datos Scielo y LILACS. Se utilizaron los descriptores disponibles en el índice de descriptores en ciencias de la salud (DECS): servicio médico de emergencia, urgencia y emergencia. Los criterios de inclusión fueron los artículos escritos en los últimos 10 años de origen nacional y en portugués, disponibles en las bases de datos o en la plataforma de la revista y respondieron a la pregunta de investigación. Resultados: Después de la búsqueda en la base de datos LILACS, utilizando los descriptores servicio médico de emergencia y emergencias, fueron encontrados 299 artículos, después de la aplicación de los filtros hubo un resultado con 18 artículos que después de la lectura de los títulos y resúmenes resultó en una muestra de 7 artículos. En la base de datos Scielo se encontraron 56 artículos, después de la aplicación de los filtros hubo un resultado con 17 artículos y con la lectura de los títulos y resúmenes se consideraron elegibles 10 artículos para la muestra. Consideraciones finales: Las principales dificultades de la atención prehospitalaria descritas en la producción científica nacional en los últimos 10 años, observadas en este estudio, fueron: falta de integración entre los servicios, demandas no pertinentes, déficit de materiales y falta de capacitación. Además, fueron citadas también: la inmovilidad en la vía pública, la exposición a la violencia urbana, la falla en la comunicación entre los servicios y la carencia de protocolos específicos de atención.

Palabras clave: Servicio médico de emergencia, Urgencia, Emergencia.

\section{INTRODUÇÃO}

Entende-se por atendimento pré-hospitalar móvel o atendimento que é prestado a vítima o mais prévio possível fora do ambiente hospitalar, composto por uma equipe multiprofissional que segue protocolos específicos de atendimento, prestando os primeiros cuidados e fornecendo um transporte seguro até ao ambiente hospitalar (BRASIL, 2003).

O atendimento pré-hospitalar é considerado de extrema relevância no contexto da assistência em urgência e emergência sendo a pioneira desses atendimentos a corporação dos bombeiros articulada com a secretaria estadual de saúde e com médicos. Entretanto os atendimentos realizados, no início dos serviços de urgência e emergência extra-hospitalar eram apenas de suporte imediato e básico, sendo os pacientes transportados sem acompanhamento de profissionais da saúde com diversas inadequações legais, especialmente no tocante à segurança (O'DWYER G, 2016).

Percebe-se que houve a necessidade de que ocorressem avanços tecnológicos, estruturais e de logística nos atendimentos especialmente em virtude das mudanças no cenário epidemiológico do país com o aumento da doenças crônico-degenerativas, ou ainda o aumento da ocorrência de problemas traumáticos associados à violência urbana e aos acidentes automobilísticos. Dados consolidados do Sistema de Informação de Mortalidade (SIM) ressaltam que a maior parte das mortes em jovens no país decorre de causas externas expressas por acidentes de transporte, agressões ou quedas (IBGE, 2016), a Portaria n. 2048 de 2002 relata que em consequência desses fatos houve um expressivo aumento nas demandas de atendimento préhospitalar móvel.

A Portaria 1.864, de setembro de 2003, considera os seguintes indicadores para acompanhamento e avalição do serviço prestado: tempo médio de resposta entre a chamada telefônica e a chegada da equipe no local da ocorrência, tempo médio decorrido no local da ocorrência, tempo médio de transporte até a 
unidade de referência, tempo médio de transporte até a unidade de referência, tempo médio de resposta total (entre a solicitação telefônica de atendimento e a entrada do paciente no serviço hospitalar de referência). Leva-se em conta outros indicadores que envolvem a regulação (porcentagem de saídas de veículos de Suporte Avançado após avaliação realizada pela equipe de Suporte Básico), taxas de mortalidade, sequelas e seguimento no atendimento hospitalar além da casuística dos atendimentos (BRASIL, 2003).

Apesar dos avanços e do crescimento da rede de urgências, o serviço ainda possui carências estruturais, a articulação para a solução destes problemas é de suma importância e pode trazer mais efetividade na rede assistencial (O'DWYER G, 2016). O apoio de gestores de diversos setores na saúde e nas redes de apoio é fundamental, formulando políticas de saúde para que se alcancem melhorias contínuas nos serviços prestados (SILVA EAC, 2017).

Para que sejam atingidos os objetivos baseados nos indicadores de qualidade de atendimento préhospitalar é fundamental o conhecimento das rotinas de atendimento, as dificuldades que podem impactar no tempo e na eficiência dos atendimentos já que estes precisam ocorrer de forma dinâmica e seguir uma ordem lógica com cada profissional exercendo seu papel. Considerando este contexto o objetivo deste trabalho foi identificar por meio de uma revisão integrativa da literatura as principais dificuldades do atendimento préhospitalar descritas na produção científica nacional dos últimos 10 anos.

\section{MÉTODOS}

Trata-se de uma revisão integrativa da literatura realizada por meio de busca nas bases de dados Scielo e LILACS. Foram utilizados os descritores disponíveis no índice de descritores em ciências da saúde (DECS): serviço médico de emergência, urgência e emergência. Os critérios de inclusão utilizados foram artigos produzidos nos últimos 10 anos de origem nacional e em língua portuguesa, disponíveis nas bases de dados ou na plataforma do periódico e que respondiam à questão de pesquisa.

Após o cruzamento dos descritores nas bases de dados selecionadas, os artigos foram selecionados conforme os critérios de inclusão através da aplicação dos filtros disponíveis. Em seguida procedeu-se a leitura dos títulos e resumos e posteriormente foi efetuada a leitura completa dos textos incluídos na amostra com a respectiva extração dos dados ou respostas para a questão de pesquisa por meio da busca no conteúdo da discussão e conclusão dos autores.

Os resultados foram então consolidados em gráficos no programa Excel do pacote Office 2013 e na forma de um quadro sinóptico, sendo possível a partir daí a definição das categorias de resposta.

\section{RESULTADOS}

Após a busca na base de dados LILACS, utilizando-se os descritores serviço médico de emergência e emergências, foram encontrados 299 artigos, após aplicação dos filtros houve um resultado com 18 artigos que após leitura dos títulos e resumos resultou em uma amostra de 7 artigos. Na base de dados Scielo foram encontrados 56 artigos, após aplicação dos filtros houve um resultado com 17 artigos e com a leitura dos títulos e resumos foram considerados elegíveis 10 artigos para a amostra. Em outro cruzamento para busca na base LILACS, com os descritores serviço médico de urgência e socorro de urgência, foram encontrados 6 resultados, sendo descartados 2 (um por não estar disponível na revista e na base de dados e outro por se tratar de uma dissertação). Ao final foram considerados elegíveis ao todo 09 artigos.

As principais dificuldades levantadas na pesquisa foram a falta de integração entre os serviços e demanda não pertinente (Figura 1), sendo a metodologia mais frequente nos estudos a qualitativa e exploratória (Figura 2). No tocante ao número de artigos observou-se maior número no ano de 2012 seguido pelo ano de 2017 (Figura 3). 
Quadro 1 - Quadro sinóptico de resultados dos artigos encontrados na base de dados LILACS e Scielo.

\begin{tabular}{|c|c|c|c|}
\hline Autor/ano & Metodologia & Número de sujeitos & $\begin{array}{l}\text { Resposta à questão de } \\
\text { pesquisa }\end{array}$ \\
\hline $\begin{array}{l}\text { VELOSO, ISC; ARAUJO, } \\
\text { MT; ALVES, M (2012) }\end{array}$ & $\begin{array}{l}\text { Estudo qualitativo do tipo } \\
\text { estudo de caso }\end{array}$ & $\begin{array}{l}31 \text { profissionais do } \\
\text { SAMU }\end{array}$ & Integração entre serviços \\
\hline $\begin{array}{l}\text { BALBINO, AC; } \\
\text { CARDOSO, MVLML, } \\
\text { (2017) }\end{array}$ & $\begin{array}{l}\text { Estudo qualitativo, com } \\
\text { caráter exploratório } \\
\text { descritivo }\end{array}$ & $\begin{array}{l}17 \text { profissionais do } \\
\text { SAMU }\end{array}$ & $\begin{array}{l}\text { Déficit de material. Falta } \\
\text { de capacitação, falta de } \\
\text { mobilidade na via pública } \\
\text { e falha de comunicação. }\end{array}$ \\
\hline $\begin{array}{l}\text { NOVACK BC et al, } \\
(2017)\end{array}$ & $\begin{array}{l}\text { Estudo qualitativo, com } \\
\text { caráter exploratório } \\
\text { descritivo }\end{array}$ & $\begin{array}{l}12 \text { profissionais do } \\
\text { SAMU }\end{array}$ & $\begin{array}{l}\text { Falta de capacitação; } \\
\text { déficit de materiais; } \\
\text { exposição à violência } \\
\text { urbana. }\end{array}$ \\
\hline $\begin{array}{l}\text { LUCHTEMBERG MN et } \\
\text { al., (2014) }\end{array}$ & $\begin{array}{l}\text { Estudo quantitativo, } \\
\text { descritivo e documental }\end{array}$ & $\begin{array}{l}1.353 .150 \text { chamadas } \\
\text { telefônicas }\end{array}$ & $\begin{array}{l}\text { Demanda não pertinente } \\
\text { e integração entre os } \\
\text { serviços }\end{array}$ \\
\hline $\begin{array}{l}\text { SANTOS MC et al., } \\
(2012)\end{array}$ & $\begin{array}{l}\text { Estudo descritivo- } \\
\text { qualitativo }\end{array}$ & $\begin{array}{l}27 \text { profissionais do } \\
\text { SAMU }\end{array}$ & Falhas na comunicação \\
\hline
\end{tabular}

\begin{tabular}{llll}
\hline $\begin{array}{l}\text { BARROS MAA et al., } \\
\text { (2013) }\end{array}$ & $\begin{array}{l}\text { Estudo documental e } \\
\text { retrospectivo, de } \\
\text { abordagem quantitativa, }\end{array}$ & $\begin{array}{c}546 \text { fichas de } \\
\text { atendimentos }\end{array}$ & $\begin{array}{l}\text { Carência de } \\
\text { planejamento e } \\
\text { protocolos específicos } \\
\text { para o idoso. }\end{array}$ \\
\hline ABREU KP et al., (2012) & $\begin{array}{l}\text { pesquisa exploratório- } \\
\text { descritiva com abordagem } \\
\text { qualitativa }\end{array}$ & 30 usuários do SAMU & $\begin{array}{l}\text { Demanda não pertinente } \\
\text { VERONESE, AM; }\end{array}$ \\
$\begin{array}{l}\text { Estudo quantitativo } \\
\text { OLIVEIRA, DLLC; NAST, } \\
\text { K, (2018) }\end{array}$ & $\begin{array}{l}26.233 \text { chamadas } \\
\text { telefônicas }\end{array}$ & Demanda não pertinente \\
\hline $\begin{array}{l}\text { ROCHA, RLP; } \\
\text { VELLOSO, ISC; ALVES, } \\
\text { M, (2009) }\end{array}$ & $\begin{array}{l}\text { Estudo de caso descritivo, } \\
\text { de natureza qualitativa }\end{array}$ & 22 profissionais de UBS & $\begin{array}{l}\text { Integração entre os } \\
\text { serviços e falha na } \\
\text { comunicação }\end{array}$ \\
\hline
\end{tabular}

Fonte: Dados da pesquisa realizada em julho de 2018.

\section{DISCUSSÃO}

A principal dificuldade apontada nos estudos analisados estava relacionada a integração entre os serviços de saúde. Como exemplo, é possível observar que as relações de poder interferem na dinâmica do atendimento de forma que a resistência por parte dos profissionais às ações de outros, gera, em alguns casos insatisfação devido ao aumento do volume de trabalho (VELOSO ISC, ARAUJO MT, ALVES M, 2012).

Afirma-se também que o processo de trabalho no atendimento pré-hospitalar deve ser realizado de forma ágil e eficaz, sendo que a demora no atendimento pelo serviço especializado pode levar à remoção por outros agentes como o Corpo de Bombeiros, Polícia Militar ou transeuntes. Um ponto importante é que essa remoção, não sendo comunicada ao Serviço de Atendimento Móvel de Urgência (SAMU), gera transtornos ao atendimento com demandas não atendidas, gastos desnecessários com o deslocamento da viatura e ao mesmo tempo revelam que não há uma comunicação ou integração entre os serviços que a fizeram (LUCHTEMBERG MN et al., 2014).

Pode haver comprometimento nas ações integradas entre os serviços quando há conflitos quanto às funções ou ao papel dos profissionais no atendimento do paciente pré-hospitalar. Nota-se este conflito no tocante às atividades da Unidade Básica de Saúde em relação ao papel do SAMU. Quando o paciente em crise aguda é admitido na UBS há problemas em estabilizá-lo por falta de estrutura e aparato tecnológico o que leva à necessidade de transferência e remoção e neste momento pode ser difícil estabelecer as ações adequadas e condutas com o paciente o que resulta em mais transtornos no processo de atendimento (ROCHA RLP, VELLOSO ISC, ALVES M, 2009). 
Figura 1 - Respostas mais frequentemente observadas nos artigos quanto às dificuldades do atendimento pré-hospitalar

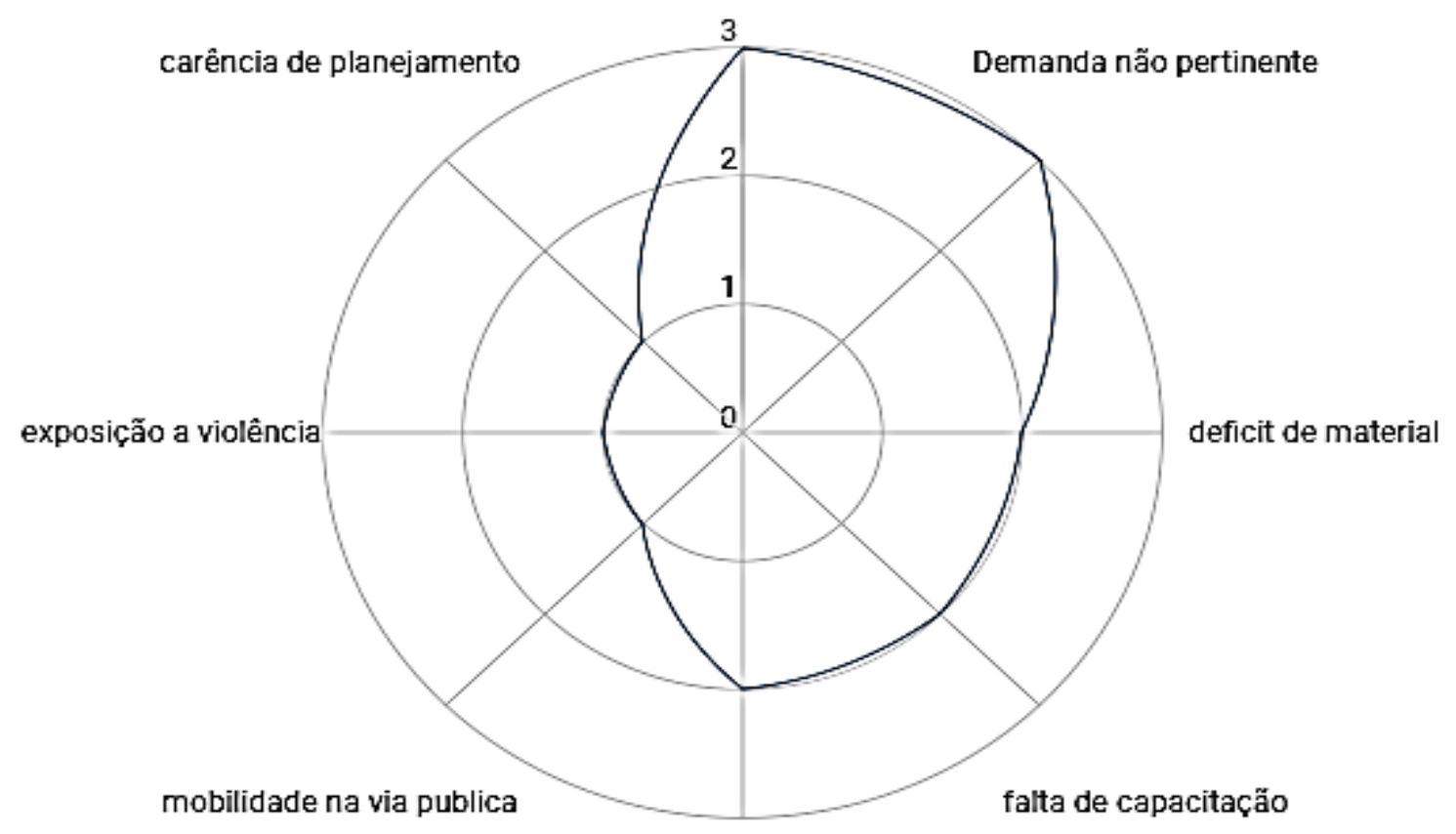

Fonte: Dados da pesquisa realizada em julho de 2018.

Figura 2 - Metodologia mais utilizada nos artigos pesquisados.

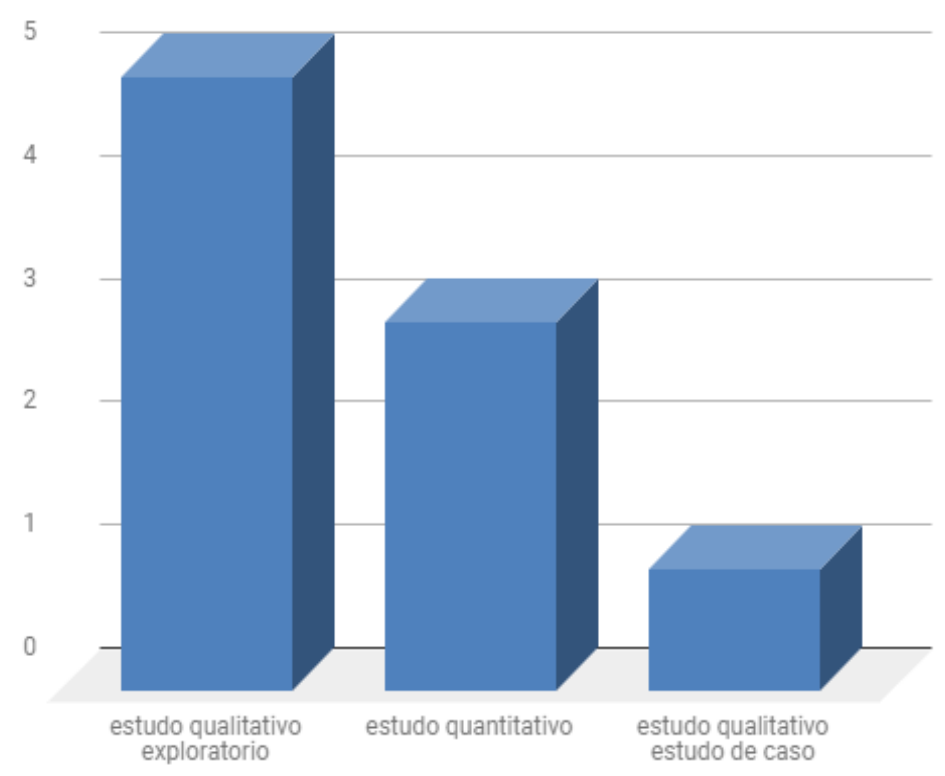

Fonte: Dados da pesquisa realizada em julho de 2018. 
Figura 3 - Número de artigos encontrados na busca de acordo com o ano de publicação.

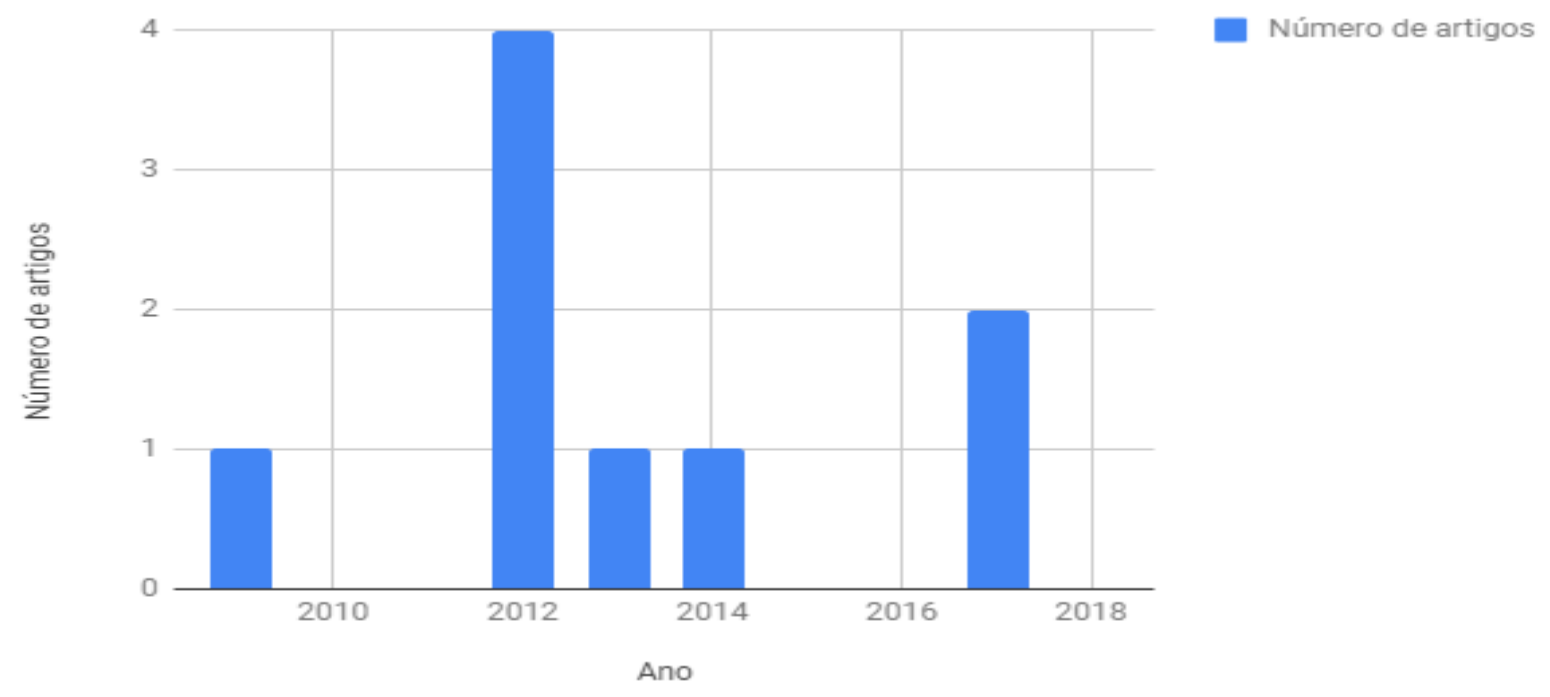

Fonte: Dados da pesquisa realizada em julho de 2018.

Entende-se por demanda não pertinente (NP) toda a situação que gera um deslocamento ou a atividade do serviço que não resulta em uma ação necessária. Neste caso citam-se os trotes, chamadas telefônicas não adequadas e outras situações que podem gerar inclusive risco para a população pela ocupação desnecessária da equipe do SAMU. Nota-se até mesmo certa sazonalidade na ocorrência das chamadas com demanda NP (VERONESE AM, OLIVEIRA DLLC, NAST K, 2018).

Há ainda a dificuldade de compreensão da população quanto a indicação do uso de ambulâncias para situações específicas relacionados ao risco de morte e agravos com maior indicação de atendimento urgente, isto pode estar relacionado ao nível de formação e diferenças de ordem social e cultural (ABREU KP et al., 2012).

Sabe-se que vários aspectos estão envolvidos na prestação de uma assistência de qualidade o que torna a questão complexa, tornando-se um grande desafio para os profissionais de saúde. A escassez de materiais é uma realidade nos atendimentos pré-hospitalares cotidianos produzindo atendimentos de baixa qualidade. Desta forma, aventa-se a necessidade de um levantamento de recursos que possa suprir essa grande demanda (NOVACK BC et al., 2017), essa perspectiva também foi mostrada nos estudos de Balbino AC e Cardoso MVLML (2017), onde a falta de recursos evidenciou o comprometimento nas transferências de pacientes entre os hospitais levando a um prolongamento na resolução do atendimento prestado.

A educação permanente, um dos métodos mais utilizados na área da saúde, visa o aperfeiçoamento do profissional na assistência ao paciente; a falta de capacitação gera transtornos nesses profissionais, pois pode comprometer o andamento dos processos durante a realização de procedimentos e ocorrências. Até mesmo profissionais mais experientes relatam dificuldades, pois a complexidade do serviço pré-hospitalar exige uma capacitação constante (BALBINO AC e CARDOSO MVLML, 2017). Novack BC et al (2017) corroboram a ideia da necessidade de qualificação dos profissionais ressaltando que é preciso intensificar as ações educativas no processo de formação dos profissionais.

O processo de comunicação é importante em todas as áreas, na saúde fornece subsídios para tomada de decisão, planejamentos, diagnósticos e segurança. Uma comunicação ineficaz pode comprometer a assistência já que os profissionais envolvidos na assistência pré-hospitalar devem fornecer informações adequadas no intuito de prestar um atendimento com qualidade e em menor tempo possível (SANTOS MC et al, 2012). 
As irregularidades nos atendimentos foram associadas ainda à carência de protocolos específicos. $O$ estudo de Barros MAA et al. (2013), no qual foram abordadas as particularidades do atendimento préhospitalar para a população idosa, refere-se que há algumas particularidades a serem consideradas visto que essas populações são mais vulneráveis a diversos agravos a saúde e portanto, carecem de assistência diferenciada (BARROS MAA et al, 2013).

\section{CONSIDERAÇÕES FINAIS}

As principais dificuldades do atendimento pré-hospitalar descritas na produção científica nacional nos últimos 10 anos, observadas neste estudo, foram: falta de integração entre os serviços, demandas não pertinentes, déficit de materiais e falta de capacitação. Foram citadas ainda, a imobilidade na via pública, a exposição à violência urbana, a falha na comunicação entre os serviços e carência de protocolos específicos de atendimento. Observa-se uma necessidade crescente de atualização dos profissionais na construção de um atendimento com técnicas adequadas e seguras e também uma abordagem mais ampla entre os serviços de saúde na rede de urgência, maior investimento em recursos humanos e materiais com disponibilidade maior dos insumos estratégicos para o atendimento assim como maior ênfase na capacitação. Outro ponto a ser considerado é a informação dos usuários e da população de modo geral quanto ao uso adequado e pertinente dos serviços de urgência evitando as chamadas que resultam em assistência desnecessária ou equivocada.

\section{REFERÊNCIAS}

1. ABREU, KP de et al. Percepções de urgência para usuários e motivos de utilização do serviço de atendimento préhospitalar móvel. Rev Gaúcha Enferm., Porto Alegre (RS) 2012. 33(2):146-152.

2. BALBINO, AC; CARDOSO, MVLML. Dificuldades no transporte inter-hospitalar de recém-nascido crítico realizado pelas equipes do serviço de atendimento móvel de urgência. Texto contexto - enferm., Florianópolis, 2017.(3)26: 18.

3. BARROS, MAA de et al. Características dos agravos e da assistência prestada aos idosos em um serviço préhospitalar móvel. Rev. enferm. UERJ, Rio de Janeiro, 2013. 1(21):569-74.

4. BRASIL. Ministério da Saúde. Política nacional de atenção às urgências. Brasília: Ministério da Saúde, 2003.

5. BRASIL. Ministério da Saúde. Portaria № 1864/GM, 2003. Brasília, DF.

6. BRASIL. Ministério da Saúde. Portaria № 2048/GM, 2002. Brasília, DF.

7. CICONET, Rosane Mortari; MARQUES, Giselda Quintana; LIMA, Maria Alice Dias da Silva. Educação em serviço para profissionais de saúde do Serviço de Atendimento Móvel de Urgência (SAMU): relato da experiência de Porto Alegre-RS. Interface (Botucatu), Botucatu, 2008. 12(26): 659-666.

8. INSTITUTO BRASILEIRO DE GEOGRAFIA E ESTATISTICA (IBGE). Síntese de indicadores sociais: uma análise das condições de vida da população brasileira: 2016. Rio de janeiro: IBGE, 2016.

9. LOPES, ACS et al. Adesão às precauções padrão pela equipe do atendimento pré-hospitalar móvel de Belo Horizonte, Minas Gerais, Brasil. Cad. Saúde Pública, Rio de Janeiro, 2008. (6)24: 1387-1396.

10. LUCHTEMBERG, MNet al. Análise de chamadas de um serviço de atendimento móvel de uma capital brasileira. Rev. Rene, 2014. (6)15: 925-32.

11. MINAYO, MCS; DESLANDES, SF. Análise da implantação do sistema de atendimento pré-hospitalar móvel em cinco capitais brasileiras. Cad. Saúde Pública, Rio de Janeiro, 2008. (8) 24: 1877-1886.

12. NOVACK, BC et al. As dificuldades vivenciadas pelos profissionais de enfermagem no atendimento pré-hospitalar. Revista Eletrônica Gestão \& Saúde, Brasília, 2017. 3(8): 1-8.

13. O'DWYER, G et al. Atenção pré-hospitalar móvel às urgências: análise de implantação no estado do Rio de Janeiro, Brasil. Ciênc. saúde coletiva, Rio de Janeiro, 2016. 7(21): 2189-2200.

14. ROCHA, RLP; VELLOSO, ISC; ALVES, M. Relações entre profissionais de uma Unidade Básica de Saúde e do Sistema de Atendimento Móvel de Urgência. Rev Med Minas Gerais. 2009. 19(4): 317-24.

15. SANTOS, MC dos et al. O processo comunicativo no Serviço de Atendimento Móvel de Urgência (SAMU192). Rev Gaúcha Enferm., Porto Alegre (RS) 2012. 33(1):69-76.

16. SILVA, EAC et al. Aspectos históricos da implantação de um serviço de atendimento pré-hospitalar. Revista Eletrônica de Enfermagem, Goiânia, 2010.3(12): 571-7.

17. VELOSO, ISC; ARAUJO, MT; ALVES, M. Práticas de poder no serviço de atendimento móvel de urgência de Belo Horizonte. Rev. Gaúcha Enferm., Porto Alegre, 2012. 4(33): 126-132.

18. VERONESE, AM; OLIVEIRA, DLLC; NAST, K. Risco de vida e natureza do SAMU: demanda não pertinente e implicações para a enfermagem. Rev. Gaúcha Enferm., Porto Alegre, 2012. 4(33):142-148. 